\title{
COMPOSIÇÃo FLORÍSTICA E ESPECTRO BIOLÓGICO DE UM CAMPO DE altitude no Parque Estadual da Serra do Brigadeiro, Minas Gerais - BrasiL ${ }^{1,2}$
}

\author{
Alessandra Nasser Caiafa ${ }^{3,4}$ \& Alexandre Francisco da Silva ${ }^{5}$
}

\section{Resumo}

(Composição florística de um campo de altitude no Parque Estadual da Serra do Brigadeiro, Minas Gerais Brasil) Nas elevadas altitudes do sudeste brasileiro são encontrados os campos de altitude. A fisionomia mais freqüentemente encontrada nos platôs relativamente extensos é a de arbustos inseridos em uma matriz de touceiras de gramíneas, com esparsas ervas e pteridófitas. Os objetivos deste trabalho foram elaborar a lista florística de plantas vasculares e determinar o espectro biológico florístico, de uma campo de altitude localizado no Parque Estadual da Serra do Brigadeiro, Minas Gerais, Brasil. Para tanto, foram realizadas expedições mensais entre julho de 2000 a janeiro de 2002. As espécies foram classificadas nas classes de formas de vida de Raunkiaer, modificadas segundo Braun-Blanquet. Para a comparação do espectro biológico da flora estudada com o espectro normal de Raunkiaer foi realizado um teste de qui-quadrado de independência. Foram coletadas 81 espécies de plantas vasculares. A família mais rica foi Orchidaceae, seguida por Asteraceae, ambas bem representadas nos campos de altitude. O elevado número de espécies exclusivas de cada sinúsia indica que as mesmas estão bem delimitadas na vegetação local. $\mathrm{O}$ espectro biológico florístico mostrou um predomínio dos hemicriptófitos, seguido pelos caméfitos, formas de vida relacionadas à fisionomia campestre. $\mathrm{O}$ teste de qui-quadrado mostrou que o espectro biológico da vegetação estudada difere do normal de Raunkiaer. É necessária, a realização de um maior número de estudos florísticos e ecológicos em campos de altitude, pois a carência atual de estudos não permite análises comparativas, ferramentas importantes para se aferir relações fitogeográficas, e para avaliar o estado de conservação das áreas possuidoras de tal formação vegetacional.

Palavras-chave: Campo de altitude, florística, espectro biológico, Parque Estadual da Serra do Brigadeiro.

\section{Abstract}

(Floristic composition of a "campo de altitude" in the Serra do Brigadeiro State Park, Minas Gerais - Brazil) In the high atitudes of Brazilian southeast, "campos de altitude" are found. The physiognomy most frequent in the relatively extensive plateaus is the one of schrubs within a matrix of bunchgrasses, with sparse herbs and pteridophytes. The aims of this paper were the elaboration of a floristic checklist of vascular plants and the determination of the floristic biological spectrum of a campo de altitude located in the "Serra do Brigadeiro" State Park, Minas Gerais - Brazil. For that, field expeditions were monthly accomplished between July 2000 to January 2002. The species were classified in the life form classes of Raunkiaer, modified according to Braun-Blanquet. A chi-square test of independence was carried out to compare the biological spectrum of the studied flora with the normal spectrum of Raunkiaer. Eighty-one species of vascular plants were identified, and the richest family was Orchidaceae, followed by Asteraceae, both well represented in "campos de altitude". The high number of species which are exclusive of each synusiae indicates that the same are well delimited in the local vegetation. The floristic biological spectrum showed a prevalence of hemicryptophytes followed by chamaephytes, life forms related to grassland physiognomy. The chi-square test showed that the biological spectrum of the studied vegetation differs from the normal of Rankiaer. The accomplishment of a higher number of floristic and ecological studies in campos de altitude is needed, since the lack of studies allows neither the realization of comparative studies, important to confront biogeographical relations nor foresight of their conservation status.

Key-words: "Campos de altitude", floristics, biological spectrum, "Serra do Brigadeiro" State Park.

Artigo recebido em 07/2004. Aceito para publicação em 03/2005.

${ }^{1}$ Parte da Dissertação de Mestrado (programa de Pós-Graduação em Botânica - UFV) do primeiro autor.

${ }^{2}$ Projeto Financiado pelo CNPq, processo $n^{\circ} 479083 / 01-0$

${ }^{3}$ Doutoranda do programa de Pós-Graduação em Biologia Vegetal - IB/UNICAMP.

${ }^{4}$ Autor para correspondência: ancaiafa@yahoo.com.br Departamento de Botânica, IB/UNICAMP, Cx. Postal: 6109, CEP: 13083-970, Campinas, SP.

${ }^{5}$ Professor Adjunto do Departamento de Biologia Vegetal - UFV. 


\section{INTRODUÇão}

Nas escarpas mais altas e íngremes das serras do sudeste brasileiro encontra-se uma vegetação predominantemente campestre, de características fisionômicas e ecológicas ímpares, denominados por Ferri (1980) campos de altitude.

Barreto (1949) ao estudar a vegetação em áreas altimontanas utilizou o termo "campos alpinos", assim como Rizzini (1963), que propôs o termo "campos altimontanos". Joly (1970) utilizou o termo introduzido por Magalhães (1966) "campos rupestres", referindo-se exclusivamente às formações sobre quartzito. Em trabalho posterior, Rizzini (1979) avançou na classificação, subdividindo, estas formações em "campos quartzíticos", para áreas sobre quartzito como as do Espinhaço, e "campos altimontanos", para àquelas sobre rochas cristalinas diversas, como os ocorrentes nas Serras do Mar e da Mantiqueira. Da mesma forma, Ferri (1980) dividiu essa formação em "campos rupestres" e "campos de altitude", e Eiten (1983), em "campo rupestre" e "campo montano", para formações sobre quartzito e sobre granito, respectivamente. Veloso (1991) classificou tal formação como "refúgios vegetacionais ou relíquias de vegetação", que segundo ele, seria toda e qualquer vegetação floristicamente diferente do contexto geral da flora dominante. Semir (1991) avançou mais ainda quando sugeriu os termos "complexos rupestres de quartzito" e "complexos rupestres de granito" para a vegetação do Espinhaço e da Mantiqueira, respectivamente, alegando que ambas as formações são rupestres, mas diferem quanto a litologia predominante, uma vez que a utilização do termo complexo permitiu particularizar todas as sinúsias vegetais associadas, como as matas nebulares, escrubes, ambientes hidromórficos, campos graminóides e os afloramentos rochosos. Mais recentemente Benites et al. (2003), sugeriram a inclusão do termo "altitude" na terminologia proposta por Semir (1991), ampliando-a para "complexos rupestres de altitude sobre granito" e "complexos rupestres de altitude sobre quartzito", por considerarem importante separar as áreas altimontanas de outros "complexos rupestres" como, por exemplo, os que ocorrem em ambientes costeiros e os que ocorrem associados à caatinga.

É importante observar que quase sempre foram feitas distinções entre os denominados campos rupestres e campos de altitude. Estas formações são fisionomicamente semelhantes, porém, diferem no que diz respeito à composição florística, as associações com outras formações vegetacionais adjacentes e principalmente, quanto à litologia predominante (Rizzini 1979; Ferri 1980; Eiten 1983, Semir 1991, Giulietti et al. 2000 e Benites et al. 2003). Além disso, diferentemente de outras formações, como a amazônia e o cerrado, não apresentam uma área nuclear de distribuição, ocorrendo em áreas disjuntas, separados por vales florestados, planaltos e bacias hidrográficas (Caiafa 2004).

Os campos rupestres ocorrem em feições mais tabulares compostas por pontões e grandes blocos rochosos desagregados, predominantemente de rochas como quartzitos e arenitos. Estão normalmente associados com o cerrado, mas também podem ocorrer associados à outras formações como caatinga (Giulietti et al. 2000). Localizam-se em modelados de rochas proteozóicas que datam $700 \mathrm{Ma}$. (pré-cambrianas), como a Cadeia do Espinhaço (ou serra Geral), dividida em dois blocos principais: a Chapada Diamantina na Bahia e a Serra do Espinhaço em Minas Gerais (Moreira \& Camelier 1977). Segundo Rizzo (1981), no estado de Goiás, os campos rupestres localizam-se, principalmente, nos pontos mais altos das serras que compõe o Maciço Goiano (Moreira 1977).

Já os campos de altitude, ocorrem sobre geoformas mais arredondadas de rochas graníticas e, ou, rochas intrusivas ácidas ricas em sílica e alumínio, e encontram-se inseridos na área de abrangência da mata atlântica (senso amplo). Localizam-se em escarpas e maciços modelados em rochas arqueanas, datadas em 3.800 Ma. (pré-cambrianas), sendo 
as de maior expressão aquelas que compõem as Serras do Mar e da Mantiqueira (Moreira \& Camelier 1977). Estima-se encontrar uma área total de aproximadamente $350 \mathrm{~km}^{2} \mathrm{de}$ campos de altitude nos cumes das Serras do Mar e Mantiqueira (Safford 1999).

Nos campos de altitude, as diferentes sinúsias de vegetação formam um mosaico, cuja fisionomiamais frequientemente encontrada nos platôs relativamente extensos é a de arbustos inseridos em uma matriz de touceiras de gramíneas, com ervas esparsas e pteridófitas (Safford 1999). Aparecem, também, como elementos da paisagem das elevadas altitudes, extensões variáveis de rocha aflorada, penhascos e picos rochosos (Safford \& Martinelli 2000).

Outra característica peculiar aos campos de altitude são as elevadas taxas de endemismo (Safford 1999). Martinelli (1996) estimou que $11 \%$ das espécies vasculares do Itatiaia são localmente endêmicas e $21 \%$ são endêmicas das áreas de campos de altitude. No Pico do Frade, Pedra do Desengano e Serra da Bocaina (Serra do Mar na porção que corta o estado do Rio de Janeiro), as porcentagens de espécies endêmicas dos campos de altitude são, respectivamente, 18, 12 e 14\% (Martinelli 1996).

Segundo Safford (1999), pesquisas básicas e aplicadas em unidades de conservação do sudeste brasileiro são raras e predominantemente restritas aos habitats de terras baixas e citou ainda, como uma das prioridades para a pesquisa e a conservação dos campos de altitude a elaboração de inventários florísticos e faunísticos destas áreas. Pode-se citar alguns trabalhos que abordam aspectos florísticos gerais (não de táxons específicos) de campos de altitude: Rizzini (1954), Dusén (1955), Brade (1956), Segadas-Vianna (1965), Martinelli (1996) e Safford (1999).

Ambientes como os campos de altitude merecem mais atenção da comunidade científica, não só pelo seu significado biológico e geológico, mas, principalmente, porque representam as primeiras áreas de drenagem para o suprimento de água de quase $25 \%$ da população brasileira, o que por si só justificaria sua preservação. (Safford 1999).
O presente trabalho teve como objetivo contribuir para o conhecimento da vegetação dos campos de altitude, por meio da elaboração de uma lista florística de plantas vasculares e da determinação do espectro biológico florístico de uma área de campo de altitude situado no Parque Estadual da Serra do Brigadeiro, em Minas Gerais.

\section{Materiais e Métodos}

Situado no Maciço da Mantiqueira, o Parque Estadual da Serra do Brigadeiro (PESB) encontra-se totalmente inserido na Zona da Mata de Minas Gerais, entre os meridianos $42^{\circ} 20^{\prime}$ e $42^{\circ} 40^{\prime} \mathrm{S}$ e os paralelos $20^{\circ} 20^{\prime}$ e $21^{\circ} 00^{\prime} \mathrm{W}$ (Fig. 1) (Engevix 1995). É constituído por rochas graníticas como migmatitos, granulitos e gnaisses granadíferos ou não e níveis eventuais de quartzo (MachadoFilho et al. 1983), apresentando relevo acidentado por escarpas e maciços com grandes áreas de rocha aflorada. De acordo com a classificação de Köeppen (1948), o clima da região é do tipo mesotérmico médio $\left(\mathrm{CW}_{\mathrm{b}}\right)$. A precipitação média anual é de $1.300 \mathrm{~mm}$ e a temperatura média anual de $18^{\circ} \mathrm{C}$ (Engevix 1995).

A vegetação do PESB é composta por fragmentos secundários de floresta estacional semidecídua (Veloso et al. 1991), da formação altimontana (Oliveira-Filho \& Ratter 1995), com campos de altitude (Ferri 1980) ocupando os platôs e as escarpas isoladas, em algumas áreas acima da cota de $1.600 \mathrm{~m}$ (Paula 1998).

Entre as muitas serras encontradas no PESB está a serra das Cabeças que é formada por três subserras, entre elas a denominada Totem Deitado. No topo desta subserra, a $1.722 \mathrm{~m}$ de altitude, existe uma área de aproximadamente seis hectares, revestida pelo campo de altitude objeto do presente trabalho.

Em que pese existirem terminologias mais detalhadas como as de Semir (1991) e Benites et al. (2003), foi adotada no presente trabalho a proposta por Ferri (1980) por ser a mais difundida até o presente momento.

O levantamento florístico relacionou as espécies vasculares diretamente associadas ao 


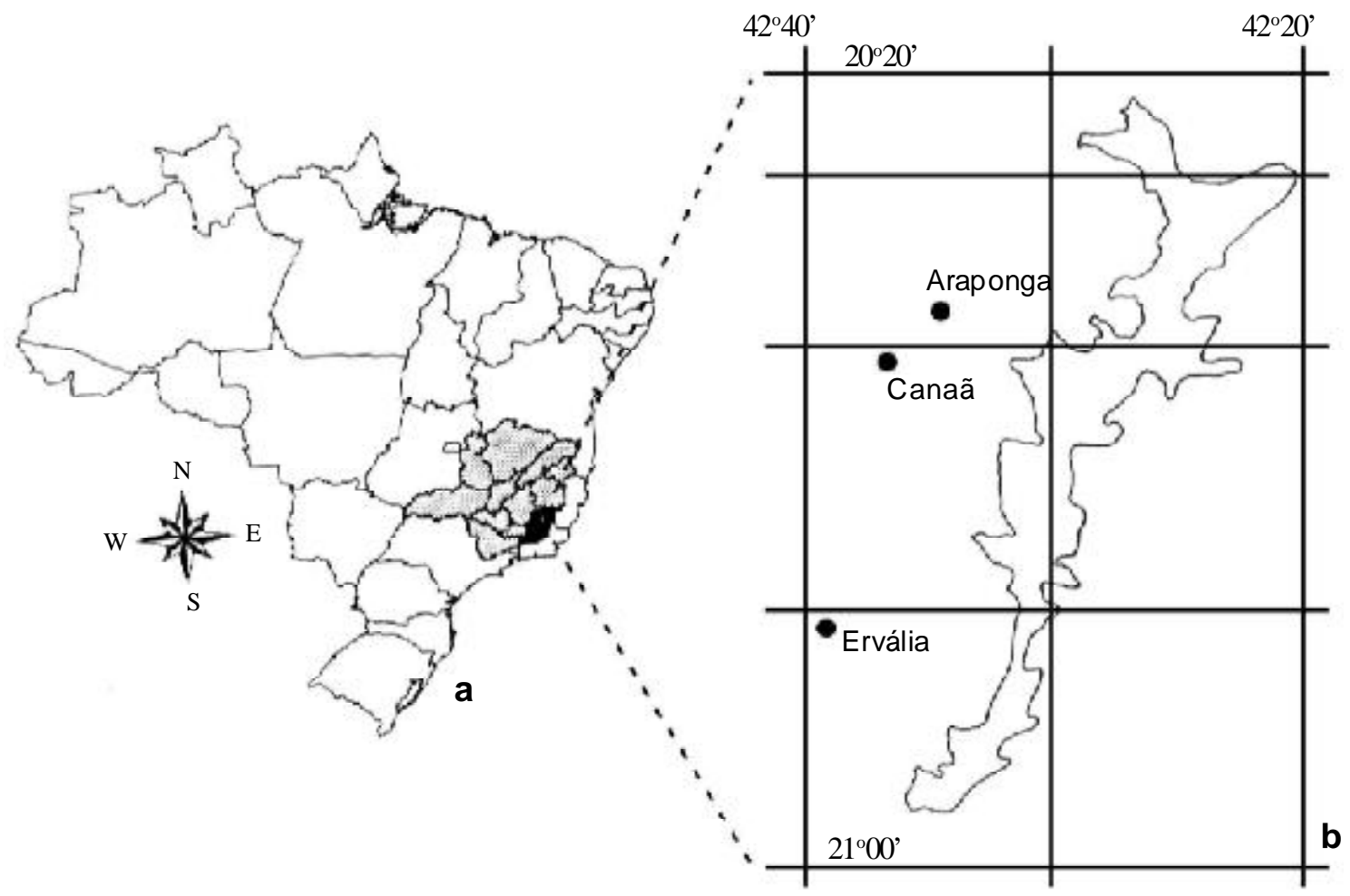

Figura 1 - Mapa do Brasil destacando o estado de Minas Gerais, a região da Zona da Mata mineira (a) e a localização do Parque Estadual da Serra do Brigadeiro - Minas Gerais (b).

afloramento rochoso, ao campo graminóide e as bordas do escrube. Para isso, foram realizadas expedições mensais para a coleta de espécimes férteis entre julho de 2000 e janeiro de 2002. O material botânico fértil foi incorporado no herbário do departamento de Biologia Vegetal da Universidade Federal de Viçosa (VIC). A identificação taxonômica foi realizada por meio de literatura especializada, por comparação mediante consultas a herbários e, quando necessário, espécimes foram enviados a especialistas.

Para a caracterização das formas de vida daflora estudada, foram consideradas as cinco principais classes de Raunkiaer para plantas adultas, revistas por Braun-Blanquet (1979). Com o intuito de se comparar o espectro biológico da flora estudada com o espectro biológico normal de Raunkiaer (Cain 1950) foi realizado um teste de qui-quadrado de independência (Beiguelman 2002), sendo necessária a aplicação do fator de correção de Yates (Beiguelman 2002). O espectro biológico foi definido segundo a maior diferença diagnosticada no teste de qui-quadrado.
Dada a falta de dados desta natureza para campos de altitude, foram realizadas comparações entre o espectro biológico aqui encontrado, com outra vegetação que apresenta certa afinidade climática e fisionômica, como o caso dos campos rupestres, sendo que para tais comparações utilizou-se os dados apresentados em Conceição \& Giulietti (2002) para um campo rupestre no Morro do Pai Inácio, Chapada Diamantina, Bahia. Foram utilizados também os dados de Ribeiro (2002) para um afloramento rochoso em campo de altitude no Parque Nacional do Itatiaia, uma sinúsia importante na composição da fisionomia e de elevada riqueza em campos de altitude.

\section{Resultados e Discussão}

Foram encontradas três sinúsias distintas. A primeira denominada escrube (Fig. 2a), formada por arbustos e arvoretas com cerca de $1,80 \mathrm{~m}$ de altura, sob as quais ocorre uma vegetação herbácea densa e variada. A segunda é composta por campos graminóides 

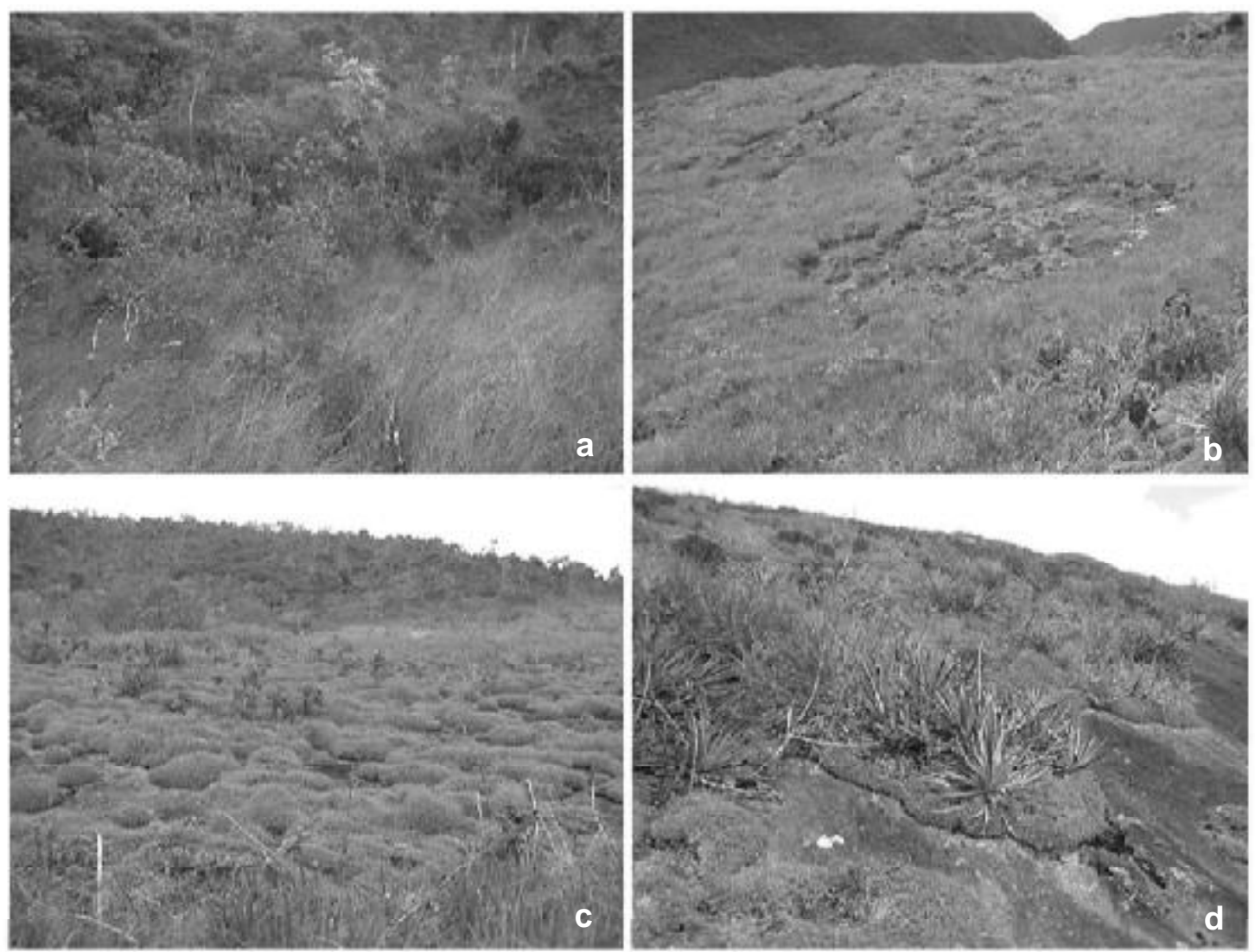

Figura 2 - a - Aspecto geral do escrube; b - Aspecto geral do campo graminóide, notar a formação de um mosaico com o afloramento rochoso; c e d - Aspecto geral do afloramento rochoso, no cume Totem Deitado, Serra das Cabeças, Parque Estadual da Serra do Brigadeiro - MG.

(Fig. 2b), onde predominam espécies de Poaceae, além de pequenos arbustos e ervas esparsos, ocupando cerca de 1,5 ha da área do cume, porém, em áreas disjuntas, formando um mosaico com a terceira tipologia que compreende o afloramento rochoso de migmatito (Figs. 2c e 2d) que apresenta grandes veios de quartzo, que onde desagregado modifica profundamente a paisagem. $\mathrm{O}$ afloramento rochoso é composto por vegetação herbáceo-subarbustiva, com no máximo $0,5 \mathrm{~m}$ de altura, disposta em ilhas de vegetação (Meirelles 1996), delimitadas por rocha nua, de formatos e tamanhos variados sobre um Neossolo Litólico Húmico (V. M. Benites comunicação pessoal), de no máximo $10 \mathrm{~cm}$ de espessura, ou, diretamente assentada sobre a rocha nua.

Foram coletadas 81 espécies, distribuídas por 60 gêneros e 31 famílias nas três sinúsias (Tabela 1). Magnoliophyta contribuiu com 75 espécies (38 Magnoliopsida e 37 Liliopsida), enquanto que Pteridophyta com apenas seis espécies. As quatro famílias mais ricas foram Orchidaceae (14 spp.), Asteraceae (12 spp.), Melastomataceae (8 spp.) e Cyperaceae (7 spp.). Segundo Safford (1999) estas famílias encontram-se bem representadas nos poucos trabalhos sobre a flora dos campos de altitude (e.g. Brade 1956).

Foram coletadas quatro novas espécies: Ditassa leonii (Fontella-Pereira \& Konno 2002) (Asclepiadaceae), uma espécie do gênero Benevidesia (Melastomataceae) e duas espécies de Eupatorium (s.l.) (Asteraceae), que ainda não foram descritas.

Observou-se que apenas duas espécies, Croton migrans (Euphorbiaceae) e Panicum sp. 2 (Poaceae), ocorreram nas três sinúsias. $\mathrm{Na}$ borda do escrube $64 \%$ das espécies são exclusivas desta sinúsia e, no afloramento 
Tabela 1 - Espécies vasculares presentes no cume Totem Deitado, Serra das Cabeças, Parque Estadual da Serra do Brigadeiro - Minas Gerais. Abreviações: $\mathrm{G}=$ geófito, $\mathrm{C}=$ caméfito, $\mathrm{H}=$ hemicriptófito, $\mathrm{T}=$ terófito, $\mathrm{N}=$ nanofanerófito, $\mathrm{M}=$ microfanerófito, $\mathrm{BE}=$ borda do escrube, $\mathrm{CG}$ = campo graminóide, $\mathrm{AH}=$ ambiente hidromórfico e $\mathrm{AR}=$ afloramento rochoso.

\begin{tabular}{|c|c|c|c|}
\hline FAMÍLIA & ESPÉCIE & ORMAS de VIDA & SINÚSIA \\
\hline ALSTROEMERIACEAE & Alstroemeria isabellana Herb. & G & $\mathrm{BE}$ \\
\hline AMARYLLIDACEAE & Hippeastrum glaucescens (Mart.) Herb. & $\mathrm{G}$ & AR \\
\hline ASCLEPIADACEAE & Ditassa leonii Fontella \& T. Konno & $\mathrm{N}$ & AReCG \\
\hline ASTERACEAE & $\begin{array}{l}\text { Achyrocline satureoides (Lam.) DC. } \\
\text { Baccharis platypoda } \text { DC. } \\
\text { Baccharis stylosa Gardner } \\
\text { Baccharis trimera DC. } \\
\text { Erigeron maximus Link \& Otto } \\
\text { Eupatorium sp. nov. } 1 \\
\text { Eupatorium sp. nov. } 2 \\
\text { Eupatorium intermedium DC. } \\
\text { Stevia claussenii Sch. Bip. ex Baker } \\
\text { Verbesina glabrata Hook. \& Arn. } \\
\text { Vernonia decumbens Gardner } \\
\text { Vernonia discolor Less. }\end{array}$ & $\begin{array}{l}\mathrm{H} \\
\mathrm{N} \\
\mathrm{N} \\
\mathrm{C} \\
\mathrm{T} \\
\mathrm{H} \\
\mathrm{H} \\
\mathrm{N} \\
\mathrm{C} \\
\mathrm{M} \\
\mathrm{N} \\
\mathrm{M}\end{array}$ & $\begin{array}{l}\text { CG } \\
\text { CGeBE } \\
\text { AReCG } \\
\text { ARe BE } \\
\text { AR eBE } \\
\text { AR } \\
\text { AReCG } \\
\text { BE } \\
\text { AR } \\
\text { BE } \\
\text { AReCG } \\
\text { BE }\end{array}$ \\
\hline BROMELIACEAE & $\begin{array}{l}\text { Dyckia bracteata } \text { (Witt.) Mez } \\
\text { Pitcairnia cf. carinata } \mathrm{Mez} \\
\text { Pitcairnia decidua } \text { L.B. Sm. }\end{array}$ & $\begin{array}{l}\mathrm{C} \\
\mathrm{H} \\
\mathrm{C}\end{array}$ & $\begin{array}{l}\text { AR } \\
\text { AR } \\
\text { AR }\end{array}$ \\
\hline BURMANNIACEAE & Burmannia bicolor Mart. & $\mathrm{T}$ & $\mathrm{AH}$ \\
\hline CYPERACEAE & $\begin{array}{l}\text { Bulbostylis scabra (Presl.) C.B.Clarke } \\
\text { Lagenocarpus comatus (Boeck.) H.Pffeif. } \\
\text { Lagenocarpus polyphyllus (Boeck.) H.Pffeif. } \\
\text { Machaerina ficticia (Hemsley) T. Koyama } \\
\text { Rhynchospora emaciata (Nees) Boeck. } \\
\text { Rhynchospora splendens Lindm. } \\
\text { Trilepis lhotzkiana Nees }\end{array}$ & $\begin{array}{l}\mathrm{T} \\
\mathrm{H} \\
\mathrm{H} \\
\mathrm{C} \\
\mathrm{H} \\
\mathrm{H} \\
\mathrm{C}\end{array}$ & $\begin{array}{l}\text { AR } \\
\text { AR } \\
\text { AR eCG } \\
\text { CG } \\
\text { AR } \\
\text { AR } \\
\text { AR }\end{array}$ \\
\hline ERIOCAULACEAE & $\begin{array}{l}\text { Leiothrix flavescens (Bong.) Ruhland } \\
\text { Paepalanthus macropodus Ruhland } \\
\text { Paepalanthus manicatus Pouls. } \\
\text { Paepalanthus sp. }\end{array}$ & $\begin{array}{l}\mathrm{H} \\
\mathrm{C} \\
\mathrm{H} \\
\mathrm{H}\end{array}$ & $\begin{array}{l}\mathrm{AH} \\
\mathrm{BE} \\
\mathrm{AR} \\
\mathrm{BE}\end{array}$ \\
\hline EUPHORBIACEAE & Croton migrans Casar. & $\mathrm{N}$ & $\mathrm{AR}, \mathrm{CGeBE}$ \\
\hline FLACOURTIACEAE & Abatia americana (Gardner) Eicher & $\mathrm{M}$ & $\mathrm{BE}$ \\
\hline GENTIANACEAE & $\begin{array}{l}\text { Hockinia montana Gardner } \\
\text { Schultesia gracilis Mart. }\end{array}$ & $\begin{array}{l}\mathrm{T} \\
\mathrm{T}\end{array}$ & $\begin{array}{l}\mathrm{AR} \\
\mathrm{AR}\end{array}$ \\
\hline GESNERIACEAE & $\begin{array}{l}\text { Siningia magnifica Otto \& Dietr. } \\
\text { Vanhouttea leonii Chautems }\end{array}$ & $\begin{array}{l}\mathrm{G} \\
\mathrm{N}\end{array}$ & $\begin{array}{l}\mathrm{AR} \\
\mathrm{AR}\end{array}$ \\
\hline IRIDACEAE & Sisyrinchium sp. & G & $\mathrm{AR}$ \\
\hline LENTIBULARIACEAE & $\begin{array}{l}\text { Utricularia sp. } 1 \\
\text { Utricularia } \text { sp. } 2\end{array}$ & $\begin{array}{l}\mathrm{T} \\
\mathrm{T}\end{array}$ & $\begin{array}{l}\mathrm{AH} \text { e } \mathrm{AR} \\
\mathrm{AR}\end{array}$ \\
\hline LOBELIACEAE & Lobelia cf. urancoma Cham. & $\mathrm{C}$ & $\mathrm{BE}$ \\
\hline LYCOPODIACEAE & $\begin{array}{l}\text { Lycopodiella camporum B. Ællg. \& P. G. Windisch } \\
\text { Lycopodium clavatum L. } \\
\text { Huperzia pungentifolia (Silveira)B. Ællg. }\end{array}$ & $\begin{array}{l}\mathrm{C} \\
\mathrm{C} \\
\mathrm{C}\end{array}$ & $\begin{array}{l}\text { CG } \\
C G \\
\mathrm{AR}\end{array}$ \\
\hline MELASTOMATACEAE & $\begin{array}{l}\text { Benevidesia sp. nov. } \\
\text { Lavoisiera imbricata (Thunb.) DC. }\end{array}$ & $\begin{array}{l}\mathrm{C} \\
\mathrm{N}\end{array}$ & $\begin{array}{l}\mathrm{AR} \\
\mathrm{BE}\end{array}$ \\
\hline
\end{tabular}




\begin{tabular}{|c|c|c|c|}
\hline FAMÍLIA & ESPÉCIE & FORMAS de VIDA & SINÚSIA \\
\hline & Marcetia taxifolia DC. & $\mathrm{N}$ & $\mathrm{BE}$ \\
\hline & Miconia theaezans (Bonpl.) Cogn. & $\mathrm{N}$ & $\mathrm{BE}$ \\
\hline & Tibouchina cf. manicata Cogn. & $\mathrm{C}$ & $\mathrm{AR}$ \\
\hline & Tibouchina sp. 1 & M & $\mathrm{BE}$ \\
\hline & Tibouchina sp. 2 & $\mathrm{~N}$ & $\mathrm{BE}$ \\
\hline & Trembleya parviflora (D. Don) Cogn. & M & $\mathrm{BE}$ \\
\hline MYRSINACEAE & Myrsine sp. & M & $\mathrm{BE}$ \\
\hline MYRTACEAE & Myrtaceae sp. & M & $\mathrm{BE}$ \\
\hline ONAGRACEAE & Fuchsia cf. regia (Vell.) Munz & $\mathrm{C}$ & CGeBE \\
\hline \multirow{14}{*}{ ORCHIDACEAE } & Cleistes gracilis Schltr. & $\mathrm{H}$ & $\mathrm{AReBE}$ \\
\hline & Epidendrum secundum Jacq. & $\mathrm{C}$ & $\mathrm{AR}$ \\
\hline & Epidendrum xanthinum Lindl. & $\mathrm{C}$ & $\mathrm{AR}$ \\
\hline & Habenaria aff. hydrophila Barb. Rodr. & $\mathrm{H}$ & $\mathrm{AR}$ \\
\hline & Habenaria janeirensis Kraenzl. & $\mathrm{H}$ & $\mathrm{AR}$ \\
\hline & Habenaria macronectar (Vell.) Hoehne & $\mathrm{H}$ & $\mathrm{AR}$ \\
\hline & Laelia sp. & $\mathrm{H}$ & $\mathrm{AR}$ \\
\hline & Oncidium barbaceniae Lindl. & $\mathrm{C}$ & $\mathrm{AR}$ \\
\hline & Oncidium blanchetii Rchb. F. & $\mathrm{C}$ & $\mathrm{AR}$ \\
\hline & Pleurothallis prolifera Lindl. & $\mathrm{H}$ & $\mathrm{AR}$ \\
\hline & Pleurothallis teres Lindl. & $\mathrm{H}$ & $\mathrm{AR}$ \\
\hline & Prescottia montana Barb. Rodr. & $\mathrm{H}$ & $\mathrm{AR}$ \\
\hline & Zygopetalum brachypetalum Lindl. & $\mathrm{C}$ & $\mathrm{AR}$ \\
\hline & Zygopetalum mackaii Hook. & $\mathrm{C}$ & $\mathrm{AR}$ \\
\hline PIPERACEAE & Peperomia galioides H.B.\& K. & $\mathrm{H}$ & $\mathrm{AR}$ \\
\hline POACEAE & $\begin{array}{l}\text { Panicum sp. } 1 \\
\text { Panicum sp. } 2\end{array}$ & $\begin{array}{l}\mathrm{H} \\
\mathrm{H}\end{array}$ & $\begin{array}{l}\mathrm{AR} \text { e CG } \\
\mathrm{AR}, \mathrm{CGeBE}\end{array}$ \\
\hline POLYGALACEAE & Polygala stricta A. St. - Hil. & $\mathrm{T}$ & $\mathrm{AR}$ \\
\hline \multirow[t]{2}{*}{ PTERIDACEAE } & Doryopteris collina (Raddi) J. Sm. & $\mathrm{H}$ & $\mathrm{AR}$ \\
\hline & Doryopteris crenulans (Fée) Christ & $\mathrm{H}$ & $\mathrm{AR}$ \\
\hline RUBIACEAE & Spermacoce poaya A. St. - Hil. & $\mathrm{C}$ & $\mathrm{AReCG}$ \\
\hline SCROPHULARIACEAE & Esterhazya splendida J.G. Mikan & $\mathrm{N}$ & $\mathrm{AR}$ \\
\hline SCHIZAEACEAE & Anemia vilosa Humb. \& Bonpl. ex Willd. & $\mathrm{H}$ & $\mathrm{AR}$ \\
\hline VELLOZIACEAE & Vellozia variegata Goethart \& Henrard & $\mathrm{C}$ & $\mathrm{AR}$ \\
\hline VELLOZIACEAE & Vellozia sp. & $\mathrm{N}$ & $\mathrm{AR}$ \\
\hline \multirow[t]{2}{*}{ VERBENACEAE } & Lantana sp. & $\mathrm{N}$ & $\mathrm{BE}$ \\
\hline & Lippia triplinervis Gardner & $\mathrm{N}$ & CGeBE \\
\hline XYRIDACEAE & Xyris filifolia L.A. Nilss. & $\mathrm{H}$ & $\mathrm{AReCG}$ \\
\hline
\end{tabular}

rochoso, $75 \%$ são exclusivas (Tabela 1). Já no campo graminóide $23,5 \%$ das espécies ocorreram apenas nesta sinúsia. Como o campo graminóide forma um mosaico com as áreas mais planas de rocha aflorada, certas espécies ali presentes, ocorreram também nas ilhas de vegetação que apresentavam maior profundidade sobre o afloramento rochoso. Entre as 17 espécies ocorrentes no campo graminóide, 10 eram compartilhadas com o afloramento rochoso (Tabela 1). A elevada proporção de espécies exclusivas de cada sinúsia indica que, no campo de altitude estudado, elas possuem composição florística distinta (ou própria). Isso corrobora com a idéia de complexos proposta por Semir (1991). Áreas ecotonais ocorreram sempre em áreas de topografia plana, as quais em certas épocas do ano (período das chuvas), apresentavam-se totalmente encharcadas e recobertas 
por uma fina camada de areia quartzoza e húmus, diretamente assentadas sobre a rocha, sendo, então, caracterizadas como pequenos ambientes hidromórficos. Entre as espécies aí encontradas destacaram-se Burmannia bicolor (Burmanniaceae), Leiothrix flavescens e Paepalanthus sp. (Eriocaulaceae), Utricularia sp. (Lentibulariaceae) e Schultesia gracilis (Gentianaceae).

A classificação das em espécies em classes de forma de vida permitiu distinguir a presença das cinco classes principais entre as propostas por Raunkiaer, modificadas por Braun-Blanquet (1979), bem como reconhecer duas subclasses de fanerófitos: nanofanerófitos e microfanerófitos. A forma de vida predominante foi a dos hemicriptófitos, seguida pela dos caméfitos e nanofanerófitos. A predominância de hemicriptófitos é um atributo relacionado às fisionomias campestres (Meirelles 1996). A alta proporção de geófitos e terófitos refletem mudanças na fisionomia da paisagem dos campos de altitude relacionadas com a sazonalidade bem marcada, como também observou Meirelles (1996) em um afloramento rochoso granítico de altitude na Pedra Grande em Atibaia (SP).

A proporção de formas de vida difere entre as sinúsias. O escrube foi a sinúsia com a maior proporção de fanerófitos (64\%), e que reuniu todos os microfanerófitos (7 spp.). Seguiram-nos os caméfitos (16\%), hemicriptófitos (12\%), terófitos e geófitos (4\% cada). No campo graminóide houve um igual predomínio de hemicriptófitos e nanofanerófitos $(35,3 \%)$ e $29,4 \%$ de caméfitos, estando ausentes os geófitos e terófitos. Já no afloramento rochoso a classe mais abundante foi a dos hemicriptófitos $(41,1 \%)$, seguida pelos caméfitos $(28,6 \%)$, terófitos e nanofanerófitos (12,5\% cada um), além dos geófitos com 5,4\%. Essas proporções de formas de vida refletem bem a fisionomia de cada sinúsia de vegetação, uma vez que é esperado um elevado número de fanerófitos na borda do escrube e uma maior proporção de hemicriptófitos no afloramento rochoso, que são as duas sinúsias mais contrastantes em termos de suas fisionomias (Figs. 2a, 2c e 2d).

$\mathrm{O}$ teste de qui-quadrado de independência (Tabela 2) demonstrou que o espectro florístico diferiu do espectro normal de Raunkiaer pelo grande número caméfitos, o que caracterizou o espectro biológico da flora estudada como camefítico. É importante salientar que o espectro normal de Raunkiaer é o resultado de um procedimento de amostragem no qual 1.000 entidades taxonômicas foram selecionadas da flora mundial de tal maneira arepresentar uma amostra randômica, admitindo-se um clima mundial homogêneo, cujos resultados para as cinco principais classes foram: fanerófitos $46 \%$, caméfitos $9 \%$, hemicriptófitos $22 \%$, geófitos $6 \%$ e terófitos $13 \%$ (Cain 1950). Segundo este mesmo autor, se o espectro normal de Raunkiaer representa com acurácia a flora mundial inteira, não éo que realmente importa, o importante é sua utilidade para ser usado como um padrão para comparações.

A comparação do espectro biológico do presente estudo com os dados apresentados para campo rupestre (Conceição \& Giulietti 2002) e um afloramento rochoso em um campo de altitude (Ribeiro 2002), demonstrou em ambos os estudos o predomínio dos hemicriptófitos (Fig. 3), além de uma expressiva importância dos caméfitos, geófitos e terófitos.

Tabela 2 - Comparação entre o espectro normal de Raunkiaer (ENR) e o espectro biológico florístico do Totem Deitado, Serra das Cabeças, Parque Estadual da Serra do Brigadeiro, Minas Gerais.

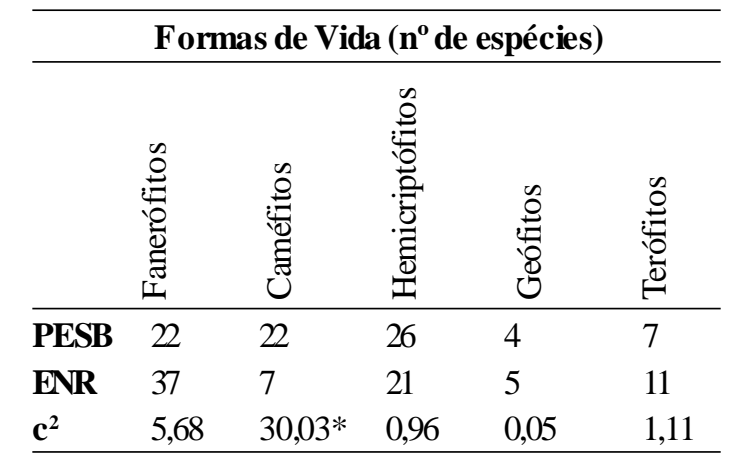

Observações: G.L. $=4 ; \mathrm{a}=0,05 ; \mathrm{c}^{2}$ tabelado $=9,488$ 


\section{Espectro Biológico - Campo de Altitude}

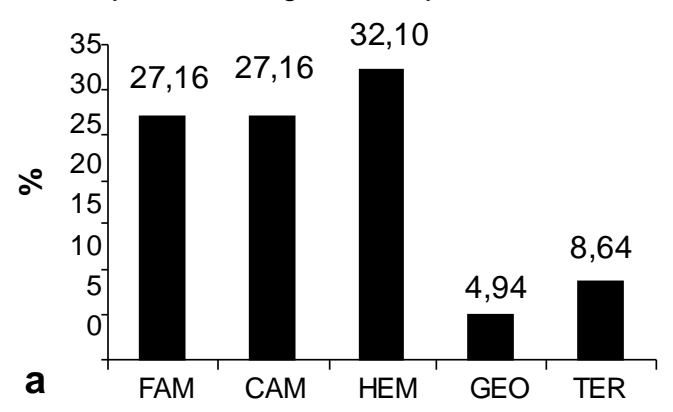

Espectro Biológico - Afloramento Rochoso
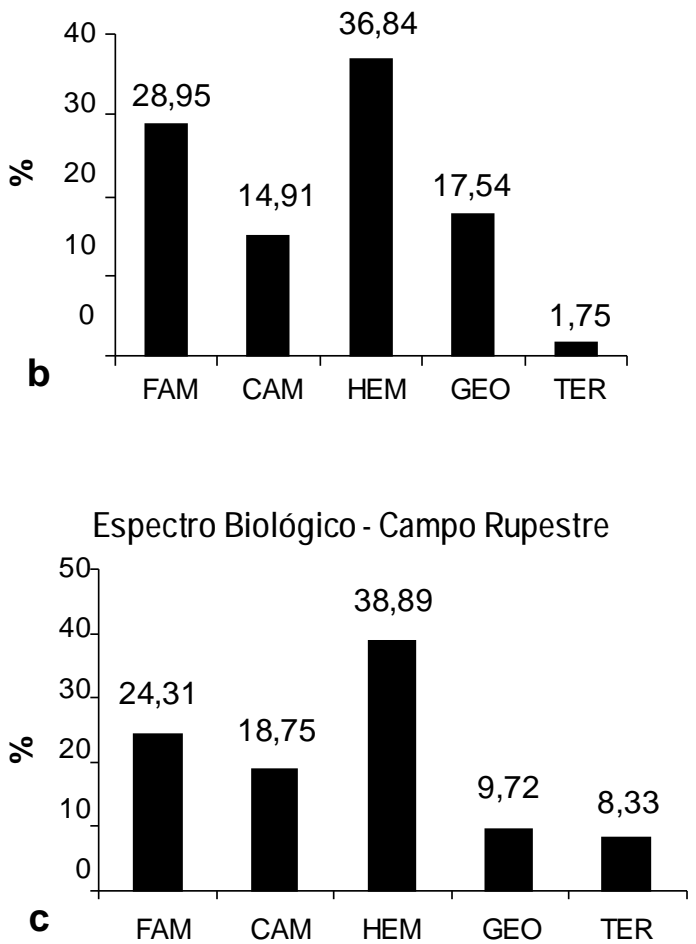

Figura 3 - Es pectros biológicos: a - campo de altitude no Parque Estadual da Serra do Brigadeiro, MG (presente estudo); $b$ - afloramento rochoso em c ampo de altitude no Parque Nacional do Itatiaia, RJ/MG (Ribeiro 2002); c - campo rupestre no Morro do Pai Inácio, Chapada Diamantina, BA (Conc eição \& Giulietti 2002). Abreviações: FAN = fanerófitos; $\mathrm{CAM}=$ caméfitos; HEM = hemicriptófitos; $\mathrm{GEO}=$ geófitos e TER = terófitos.
O afloramento rochoso do Itatiaia diferiu dos demais na proporção de terófitos (Fig. 2b), o que segundo Ribeiro (2002) pode ser explicado pela dificuldade que uma planta pode ter em completar seu ciclo de vida durante uma estação, quando há conjunção de baixas temperaturas e escassez de solos e nutrientes.

Em regiões de alta variação térmica entre o dia e a noite, e sazonalidade bem marcada, como nos campos de altitude e campos rupestres, os hemicriptófitos e os caméfitos parecem ser as formas de vida mais apropriadas a estes ambientes, pois na época de condições climáticas desfavoráveis (inverno, a estação seca) suas gemas encontram-se protegidas ao nível do solo e/ou pelas escamas, folhas ou bainha das folhas já secas da estação passada.

\section{CONSIDERaÇões FinaIS}

Apesar dos campos de altitude serem frequientes nas paisagens do sudeste brasileiro, são necessários um maior número de estudos florísticos e ecológicos sobre esta formação vegetacional, pois só assim será possível a realização de estudos comparativos tão importantes para se aferir as efetivas relações fitogeográficas, ecológicas e fisionômicas, além de permitir se avaliar com maior segurança o estado de conservação desta formação vegetacional frágil, ímpar e de grande beleza cênica.

\section{Agradecimentos}

Ao $\mathrm{CNPq}$ e à CAPES pela bolsa de mestrado concedida; ao IEF-MG pela licença para a execução do trabalho no Parque Estadual da Serra do Brigadeiro; a todos os especialistas que auxiliaram na identificação de espécies; aos Doutores Cláudio Coelho de Paula e João Augusto Alves Meira Neto, pelas sugestões na fase da elaboração da dissertação, e aos revisores anônimos deste manuscrito cujas sugestões e correções foram de grande valia para o aprimoramento do mesmo. 


\section{REFERÊNCIAS BIBLIOGRÁFICAS}

Barreto, H. L. 1949. Regiões fitogeográficas de Minas Gerais. Boletim de Geografia 14: 14-28.

Benites, V. M., Caiafa, A. N., Mendonça, E. S., Schaefer, C. E. \& Ker, J. C. 2003. Solos e Vegetação nos Complexos Rupestres de Altitude da Mantiqueira e do Espinhaço. Floresta e Ambiente 10(1): 76-85.

Beiguelman, B. 2002. Curso Prático de Bioestatítica. FUNPEC: Ribeirão Preto. 274p.

Brade, A. C. 1956. A flora do Parque Nacional do Itatiaia. Boletim do Parque Nacional do Itatiaia 5: 1-112.

Braun-Blanquet, J. 1979. Fitosociología: bases para el estudio de las comunidades vegetales. Blume. Madrid. 820p.

Cain, S. A. 1950. Life-Forms and Phytoclimate. Botanical Review 16(1): 1-32.

Caiafa, A. N. 2004. Unidades de conservação em Campos de Altitude e Campos Rupestres. Novo Disc Mídia Digital: Viçosa. (Cd-Rom dos simpósios, palestras e mesas redondas, ocorridos no $55^{\circ}$ Congresso Nacional de Botânica e $26^{\circ}$ Encontro Regional de Botânicos de MG, BA e ES).

Conceição, A. \& Giulietti, A. M. 2002. Composição florística e aspectos estruturais de campo rupestre em dois platôs do Morro do Pai Inácio, Chapada Diamantina, Bahia, Brasil. Hoehnea 29(1): 37-48.

Dusén, P. K. H. 1955. Contribuições para aflora do Itatiaia. Boletim do Parque Nacional do Itatiaia 4: 6-88.

Eiten, G. 1983. Classificação da vegetação do Brasil. CNPq/Coordenação Editorial: Brasília. 305p.

Engevix. 1995. Caracterização do meio físico da área autorizada para criação do Parque Estadual da Serra do Brigadeiro Relatório técnico final dos estudos - 8296RE-H4-003/94 "VER. 1". Instituto Estadual da Floresta, BIRD/PRÓFLORESTA/SEPLAN. 34p.
Ferri, M. G. 1980. Vegetação Brasileira. Editora da Universidade de São Paulo: São Paulo. 157p.

Fontella-Pereira, J. \& Konno, T. U. 2002. Estudos em Asclepiadaceae XXXI Duas novas espécies de Ditassa para o Brasil. Bradea 8(47): 319-322.

Giulietti, A. M., Harley, R. M., Queiroz, L. P., Wanderley, M. G. L.\& Pirani, J. R. 2000. Caracterização e endemismos nos Campos Rupestres da Cadeia do Espinhaço. Tópicos Atuais em Botânica. XLI Congresso Nacional de Botânica, Brasília, Distrito Federal. p. 311-318.

Joly, A. B. 1970. Conheça a vegetação brasileira. Editora da Universidade de São Paulo e Polígono. São Paulo. 165p.

Koeppen, W. 1948. Climatologia. Fondo Cultura Económica. México \& Buenos Aires. 478p.

Machado-Filho, L., Ribeiro, M. W., Gonzalez, S. R., Schenini, C. A., Santos-Neto, A., Palmeira, R. C. B., Pires, J. L., Teixeira, W. \& Castro, H. E. F. 1983. Geologia. In: Projeto RADAMBRASIL. Geologia. Folhas SF.23/24 Rio de Janeiro/Vitória. Volume 32. Rio de Janeiro. p. 56-66.

Magalhães, G. M. 1966. Sobre os cerrados de Minas Gerais. Anais da Academia Brasileira de Ciências 38(supl.): 59-70.

Martinelli, G. 1996. Campos de Altitude. Editora Index. Rio de Janeiro. 160p.

Meirelles, S. T. 1996. Estrutura da comunidade e características funcionais dos componentes da vegetação de um afloramento rochoso em Atibaia - SP. Tese (Doutorado em Ecologia e Recursos Naturais) Universidade Federal de São Carlos, São Carlos, SP. 270p.

Moreira, A. A. N. 1977. Relevo. In: Geografia do Brasil: Região Centro-Oeste. Fundação Instituto Brasileiro de Geografia e Estatística, Rio de Janeiro, v. 4, p. 1-34.

Moreira, A. A. N. \& Camelier, C. 1977. Relevo. In: Geografia do Brasil: Região Sudeste. Fundação Instituto Brasileiro de Geografia e Estatística, Rio de Janeiro, v. 3, p. 1-50. 
Oliveira - Filho, A. T. \& Ratter, J. A. 1995. A study of the origin of Central Brazilian Forests by the analysis of plant species distribution patterns. Edinburgh. Journal of Botany 52(2): 141-194.

Paula, C. C. 1998. Florística da família Bromeliaceae no Parque Estadual da Serra do Brigadeiro, MG - Brasil. Tese (Doutorado em Botânica) - Universidade Estadual Paulista, Rio Claro, SP. 238p.

Ribeiro, K.T. 2002. Estrutura, dinâmica e biogeografia de ilhas de vegetação rupícola do Planalto do Itatiaia, RJ. Tese (Doutorado em Ecologia) - Universidade Federal do Rio de Janeiro, Rio de Janeiro, RJ. 116p.

Rizzini, C. T. 1979. Tratado de fitogeografia do Brasil. Ed. HUCITEC: São Paulo. 374p.

Rizzini, C. T. 1963. Nota prévia sobre a divisão fitogeográfica do Brasil. Separata da Revista Brasileira de Geografia, 1. Rio de Janeiro: Instituto Brasileiro de Geografia Estatística. 64p.

Rizzini, C. T. 1954. Flora Organensis. Arquivos do Jardim Botânico do Rio de Janeiro 13: 118-243.
Safford, H. D. 1999. Brazilian Páramos I: An introduction to the physical environment and vegetation of the campos de altitude. Journal of Biogeography 26: 693-712.

Safford, H. D., \& Martinelli, G. 2000. Southeast Brazil. In: Inselbergs: Biotic diversity of isolated rock outcrops in Tropical and Temperate regions. Alemanha: Springer, p. 339-389.

Segadas-Vianna, F. 1965. Ecology of the Itatiaia Range, southeastern Brazil I. Altitudinal zonation of vegetation. Arquivos do Museu Nacional 53: 7-30.

Semir, J. 1991. Revisão taxonômica de Lychnophora Mart. (Vernoniae: Compositae). Tese (Doutorado em Biologia Vegetal), Universidade Estadual de Campinas, Campinas, SP. v. 2, p. 273-515. Veloso, H. P.; Rangel Filho, A. L. R.; Lima, J. C. A. 1991. Classificação da vegetação brasileira, adaptada a um sistema universal. Rio de Janeiro: IBGE, Departamento de Recursos Naturais e Estudos Ambientais. 123p. 

